micropenis, and distinct anomalies of the hands and feet. Another example of Wiedemann syndrome? Clin Genet Aug 1994;46:205-208). (Respond: Professor NC Nevin, Department of Medical Genetics, Floor A, Lisburn Road, Belfast BT9 7AB, UK).

COMMENT. Wiedemann and associates (1985) described 2 males, first cousins, with similar characteristics, whose mothers and maternal grandfather had short broad thumbs and halluces. The present case, the second report of this syndrome, had normal parents. Autosomal or Xlinked dominant inheritance was thought to be most consistent with the findings.

\title{
LIFE EXPECTANCY WITH CEREBRAL PALSY
}

The life expectancy of children with idiopathic cerebral palsy born during 1966-84 to mothers resident in Mersey region has been analysed at the Department of Public Health, University of Liverpool, UK. Among 1251 subjects traced, one third had quadriplegia, one third hemiplegia, a quarter had diplegia, and the remainder dyskinesia and ataxia. A quarter had severe ambulatory disability, a fifth severe manual disability, and a third an IQ of 50 or less; $11 \%$ had died. One third had a birth weight of $2500 \mathrm{~g}$ or less, and one third a gestational age of 37 weeks or less. Those with normal birth weight had the highest proportion of severe disabilities. About $85-90 \%$ of subjects with CP survived to 20 years compared to $97 \% 20$ year survival in the general population in 1970-2. Subjects with mild functional disabilities (ambulation, manual dexterity, and IQ deficits) had 20 year survival of $99 \%$, while those severely disabled had a 50\% 20 year survival. Birth weight and gestational age were less predictive of survival than functional disability. (Hutton JL et al. Life expectancy in children with cerebral palsy. BMI 13 August 1994;309:431435). (Respond: Professor Peter OD Pharoah, Department of Public Health, University of Liverpool, Liverpool L69 3BX, UK).

COMMENT. One-half of severely disabled cerebral palsied children survived to age 20 , and the life expectancy to 20 years of mild to moderately disabled children was not much lower than that of unaffected children. As more severely affected low birth weight infants survive with advances in neonatal care, future cohorts may show a higher proportion of severely disabled with $\mathrm{CP}$ and a life expectancy approaching that of the normal birth weight CP children. The social, educational, health service, and medico-legal aspects of these findings are noted. A much shorter life expectancy is reported in US studies.

\section{BRAIN DEVELOPMENT MEASURED BY MRI}

MRI data read as normal on 88 male and female patients aged 3 months to 30 years and on 73 healthy male volunteers aged 21 to 70 years were quantified and the volumes of cortical white matter, gray matter, and CSF were computed in a study at the Department of Veterans Affairs Medical Center, and the Department of Psychiatry and Behavioral Science, Stanford University School of Medicine, CA. In the younger samples, obtained from four California clinics, intracranial volume increased by about $300 \mathrm{ml}$ from 3 months to 10 years. Head size of boys was larger than that of girls by about $70 \mathrm{~mL}$, but both sexes followed the same growth trend. Cortical gray matter volume peaked at age 4 years and decreased thereafter; cortical white matter volume increased steadily until age 20 years: cortical and ventricular CSF volumes remained constant. In the older sample, cortical gray matter volume decreased 